\title{
Mainstreaming medical humanities in continuing professional development and postgraduate training
}

\author{
Authors: Desmond O'Neill, ${ }^{A}$ Brendan Kelly, ${ }^{B}$ Shaun O'Keeffe ${ }^{C}$ and Hilary Moss ${ }^{D}$
}

\begin{abstract}
Although the medical humanities are increasingly well established in terms of scholarly research and undergraduate medical education, a notable deficit has been its low profile in postgraduate training and continuing professional development (CPD).

We undertook to develop a programme in the medical humanities to address the deficit in CPD and specialist training, working with colleagues in a range of colleges and disciplines in Ireland. We describe the first element, a masterclass embedded in a series devoted to more traditionally recognised clinical aspects of CPD, webcast live to a range of hospitals across Ireland, whose focus was an introduction to the medical humanities by a panel of clinician scholars each of whom had published in peer-reviewed journals on the topic.

Postgraduate colleges of medicine can play an important role in developing medical humanities strands in their postgraduate training and CPD activities. Given the relative novelty of developing postgraduate training and CPD in medical humanities, it may be worthwhile developing an intercollegiate platform among the wider range of medical colleges in the British Isles to jointly develop the most effective mechanisms to further propagate medical humanities and leverage the expertise of a larger pool of faculty in medical humanities.
\end{abstract}

KEYWORDS: Medical humanities, continuing professional development, postgraduate training

DOI: 10.7861/clinmed.2019-0332

And so each venture / Is a new beginning, a raid on the inarticulate TS Eliot, East Coker ${ }^{1}$

\section{Introduction}

Although the medical humanities are increasingly well established in terms of scholarly research and undergraduate

Authors: ${ }^{\text {A }}$ director of the National Office for Traffic Medicine, Royal College of Physicians of Ireland, Dublin, Ireland; ${ }^{\text {B }}$ professor of psychiatry, Trinity College Dublin, Dublin, Ireland; ' $p$ rofessor and consultant geriatrician, Galway University Hospitals, Galway, Ireland;

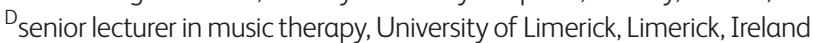

medical education, a notable deficit has been its low profile in postgraduate training and continuing professional development (CPD). ${ }^{2,3}$ Although there is a movement to relabel medical humanities as either health humanities or medical and health humanities to signify the broader range of healthcare disciplines engaged with the venture, both major journals in the field are still labelled as medical humanities, and we use the term on the understanding that it is inclusive of all healthcare disciplines. Given the key role of the medical humanities in the broader knowledge base of healthcare in terms of widening our understanding of what it is to be human, well or ill, and to experience the healthcare system, it is important that advances and developments in the topic are incorporated into professional development and training. ${ }^{4}$ The benefits of such an approach apply across the professional working life as many older clinicians may not have had access in their undergraduate programmes to the more formalised approach to the topic currently in place. In addition, the provision of medical humanities CPD and its incorporation into postgraduate training should prompt development of clinical faculty and act as a portal of entry for more clinicians to engage with reflection and scholarship in the humanities. This is all the more important in terms of an increasing separation between clinician scholars active in the field and those from the arts and humanities: a recent study confirmed this divide with little indication of joint working between both groups of disciplines. ${ }^{5}$

The range of interests and disciplines involved is large, including but not confined to literary and film studies, theatre, music, history, philosophy, ethics, and arts and health. Ethics is the one element often addressed in postgraduate training, if to a lesser extent in CPD. However, our interest group in the medical humanities in the Royal College of Physicians of Ireland (RCPI) could not detect any CPD activities in the medical colleges in UK, North America or Oceania that had promoted the broad range of medical humanities in a manner similar to updates in clinical topics. We undertook therefore to develop a programme in the medical humanities to address the deficit in CPD and specialist training, working with colleagues in a range of colleges and disciplines in Ireland including medicine, psychiatry, obstetrics and gynaecology, general practice, and music therapy. The first element was to provide a masterclass in a series devoted to many aspects of CPD, webcast live to a range of hospitals across Ireland whose focus was an introduction to the medical humanities by a panel of clinician scholars each of whom had published in peerreview journals on the topic. 


\section{Format of masterclass}

The introductory talk gave an overview of medical humanities, ranging from definitions and significance to its long history, as well as underlining that medicine was also one of the humanities; in the words of a modern pioneer, Edmund Pellegrino, 'Medicine is the most humane of the sciences, the most empiric of arts, and the most scientific of the humanities. ${ }^{6}$ As well as outlining the many resources available, including the space accorded to humanities in many generalist and specialist journals and most notably in the Perspectives section in the Lancet, the introduction touched on the challenges and opportunities of interdisciplinary working with scholars in the arts and humanities. ${ }^{7}$

\section{History, law and medicine}

The imperative for doctors to engage with historians in the history of medicine is compelling. In 1980, an unsigned editorial, provocatively titled 'Medical history without medicine', appeared in the Journal of the History of Medicine and Allied Sciences. ${ }^{8}$ The editorial noted that, prior to 1945 , most medical historians, whether amateur or professional, were doctors. Between 1945 and 1980, however, the number of professional medical historians grew and came to include a large proportion with little or no medical or scientific training. The new medical historians were, increasingly, students of history at universities rather than medical doctors in clinical practice.

The 1980 editorial highlighted the fact that academic seminars in departments of history, despite their usefulness, were far removed from the clinic and from the practical problems of medical practice. As a result, medical history focused increasingly on medicine as an aspect of cultural or social history. This, in the view of the editorial, provided an incomplete and distorted picture of the history of medicine and, especially, the history of the practice of medicine. While cultural and social histories were fascinating in their own right, they did not explore basic medical sciences and clinical medicine. What emerged, then, was 'medical history without medicine', whereby medicine was seen only in cultural and social, rather than clinical, terms.

The 1980 editorial overstated its case with admirable rhetorical skill, but its argument was not without basis: doctors can, in ideal circumstances, bring a unique sensibility and awareness to bear on the history of medicine, once their medical knowledge and clinical experiences are coupled with training in historical analysis. In 1994, Roy Porter and Mark S Micale provided a fascinating discussion of the position of the psychiatrist-historian in this context and noted that many influential historical interpreters of psychiatry in the previous century were trained as physicians and practised psychiatry alongside their work as historians. ${ }^{9}$ Both roles are complementary, once undertaken with knowledge, training and humility.

In 2005, John C Burnham, in What is medical history?, argued that there are five key 'dramas' in the history of medicine, relating to the histories of the healer; the sick person; various diseases; discovery and communication of knowledge; and interactions between medicine and health on the one hand and society on the other. ${ }^{10}$ Clearly, an increasing focus on cultural and social history alone will fail to address many of the 'dramas' correctly identified by Burnham.

A similar argument applies to the relationship between medicine and law, which is one of the neglected humanities in this context, possibly because law is often considered to be a social science rather than a humanities subject, or to stand on its own outside such categorisations. But any practising doctor who has experience of the application of medical law in day-to-day clinical practice will readily appreciate that medical law demonstrates features of both the humanities and the social sciences, as well as its own un-categorisable characteristics.

More specifically, if 'humanities' are defined as subjects concerned with human societies and culture, then medical law is undoubtedly a humanities subject, not least because the chasm between the theory of law and its application is mediated, in large part, by society and culture. ${ }^{11}$ For medical law, this is arguably most evident in psychiatry, the branch of medicine in which legislation plays the greatest role in day-to-day practice, owing to the occurrence of involuntary care. ${ }^{12}$ The roles of society and culture are vital in this context.

To take a recent example, India's Mental Healthcare Act, 2017 came into effect on 29 May 2018 and granted a legally binding 'right to access mental healthcare and treatment' to India's population of 1.3 billion people, which is one-sixth of the planet's population. ${ }^{13}$ This is a fully justiciable right, which can be pursued in the courts, but what it really means in practice is very unclear. Despite its apparently progressive measures, some psychiatrists in India argue that the new legislation is inconsistent with Indian culture and values, and might, in fact, impede the provision of much-needed mental healthcare. ${ }^{14}$ Clearly, implementation will require careful knowledge of relevant cultures if the potential of the legislation is to be realised in practice.

These complex clinical issues benefit greatly from careful consideration of humanities such as history and law in the context of day-to-day clinical practice and policy development. As a result, history, law and other humanities richly merit places in CPD programmes for clinicians both in order to ensure that doctors in clinical practice benefit from knowledge of the humanities and in order to ensure that the humanities remain connected with the realities of clinical care.

\section{Literature and ethics}

The teaching, and sometimes practice, of medical ethics provides an example of one criticism of modern medicine - that it often follows a philosophy of 'reductionism', of deconstructing the complex phenomena of illness and health and of being a patient (or a doctor) into overly simple components. ${ }^{15}$

Teaching bioethics in recent decades has been dominated by the simplicities of 'principlism', described by Harris as 'cookbook ethics.' ${ }^{16}$ In a highly influential book, Beauchamp and Childress argued that analysis of four principles (respect for autonomy, non-maleficence, beneficence and justice) provides a useful framework for thinking about ethical problems and deciding what to do. ${ }^{17}$ Much of the discourse around ethical dilemmas often reduces further to a simple tussle between respect for autonomy (recast as patient-centred care) and beneficence (labelled as paternalism) and one where there is a clear victor: 'the triumph of autonomy in bioethics'. ${ }^{18}$

Oversimplification of ethical dilemmas has also been criticised by Nash who noted that the traditional summary case report used in teaching emphasise action over characterisation and eliminate most of the nuance and motivation for people's behaviour and attitudes. ${ }^{19}$ In contrast, he suggests that close reading of short stories supports engagement with the messiness and ambiguity of real life and the need to attend to detail and to different perspectives and biases. A similar point was made by Terry and Gogel who noted literature's value for encouraging 'the cultivation of empathy for the suffering; the puzzling over seemingly incommensurable values placed on human lives; the coming to terms with human frailty, failure, and morality. ${ }^{20}$

Human frailty is not just a property of patients, of 'them'. Checkhov's doctors, for example, show the full range of medical virtues and vices, from kindness and altruism to insensitivity and 
incompetence. ${ }^{21}$ Insight into the failings of doctors is not restricted to clinician writers: the gradual loss of idealism of Dr Lydgate in George Eliot's Middlemarch - which might now be regarded as an example of burnout - is a cautionary tale for young doctors. ${ }^{22}$

There are some traps of course. Studying the humanities will not result in 'remedial humanisation' or make doctors into better people: a recent review of the, often skilled, poetic output of major tyrants is a useful corrective to this attitude. ${ }^{23,24} \mathrm{~A}$ focus on, often 'highbrow', literature ignores the learning (and fun) to be gained from other branches of the humanities. ${ }^{25,26}$ 'Medical humanities' encompass a broad and often ill-defined area of academic and educational pursuits (although this may be a strength rather than a weakness).

Medical ethics is inherently rich and complex. In recent editions of their book, Beauchamp and Childress have rejected the misuse of their principles, noting instead the need in moral reasoning to 'blend appeals to principles, rules, rights, virtues, analogies, paradigms, parables and interpretations. ${ }^{27}$ The value of the medical humanities in bioethics may be in providing clinicians with a greater and richer store of such parables.

\section{Arts and health}

Arts and health practice is defined as arts-based activities that aim to improve individual and community health and healthcare delivery and which enhance the environment by providing artwork or performances. ${ }^{28}$ The field of arts in healthcare embraces a wide range of practices including medical humanities; design aspects of healthcare; arts in hospice / end-of-life care; arts therapies; and arts and ageing, and has attracted increasing engagement by clinicians. ${ }^{29,30}$

This field, however, can suffer from over-zealous and unsubstantiated claims of effectiveness, a perception that engagement in the arts is only for the minority elite, and financial barriers given the urgent priorities for healthcare spending. ${ }^{31,32}$ Nevertheless, a body of work is well established that attests to the importance of creative engagement and access to the arts in improving the aesthetic environment of healthcare spaces and as a vehicle for self-expression. ${ }^{33}$

Hospitals are arguably an aesthetically neglected environment. Strategic use of the arts can create a sense of welcome, choice, control and beauty conducive to improved health and wellbeing. ${ }^{34,35}$ Similarly, the arts can be used to illuminate the experience of the person who is ill (sometimes literally giving a non-verbal client a language through which to express themselves) and support clinicians in expressing or reflecting on their own experiences. The broad range of films and novels on health themes attests to the power of the arts as a means of reflection on what it is to be human and to be ill. ${ }^{36,37}$ The processes for ensuring congruence between arts and health and clinical care and environments is as yet relatively unformed but ideally should seek to better understand the arts, culture and leisure engagement of patients: helpful guidance on curatorship in arts and health is emerging. ${ }^{32,38}$

Examples of such work through engagement of doctors, nurses and patients with an arts director and range of artists in Tallaght University Hospital in Dublin include a triptych of new compositions by composer Ian Wilson, reflecting on the major illnesses of older age; a mental health film festival where each film was followed by discussion facilitated by a psychiatrist and a film maker; a mural project in the paediatric unit and a hospital workplace choir for staff.

Another positive development has been an arts and health research cluster established in the University of Limerick with 40 members from every faculty across the university, bound together by an interest in arts and health. A bridging of the perceived gap between medicine and the arts is welcome, which will necessarily require accredited training for artists working in healthcare spaces and a recognition of the need to fund and support creativity and arts engagement in healthcare spaces. ${ }^{31}$ A particular opportunity arises from synergy with arts and music therapists: although there needs to be clear recognition of the very different roles, training and goals of arts and healthcare practitioners and art and music therapists, there is much to be learned from sharing the research and practice in each sphere of engagement.

\section{Discussion}

As outlined in this introductory masterclass, the breadth and variety of the medical humanities is simultaneously an opportunity and a challenge for clinicians. Harnessing the insights of the many forms of arts and humanities can deepen our understanding of the practice of medicine, the authenticity of experiences of patients, families and healthcare professionals, and sustain acceptance of ambiguity and uncertainty in clinical practice. More challenging is the development of a shared professional articulacy in the discourse of the medical humanities and the development of clinical faculty to engage meaningfully with scholars in the arts and humanities departments who are active in medical humanities.

Postgraduate colleges of medicine can play an important role in developing medical humanities strands in their postgraduate training and CPD activities. In the RCPI, further development in the programme for 2019/20 include masterclasses on medical writing and 'slam' storytelling, elective modules in narrative medicine higher specialist training, and work with the Abbey Theatre on communication with trainees in obstetrics and gynaecology (https://courses.rcpi.ie/catalog? pagename $=$ Medical-Humanities) .

Given the relative novelty of developing postgraduate training and CPD in medical humanities, it may be worthwhile developing an intercollegiate platform among the wider range of medical postgraduate colleges in the British Isles to jointly develop the most effective mechanisms to further propagate medical humanities and leverage the expertise of a larger pool of faculty in medical humanities, often relatively modest in any one college or academic centre. Among the elements which may support this, arising from our experiences, is an emphasis on high scholarly standards and professional recognition through promoting publication in peer-reviewed journals as an expected standard in the selection of clinical faculty for CPD activities, and to the greatest extent possible that such faculty members are in active clinical practice. Broadening the scope beyond medical colleges to centres of professional development of other healthcare professions, from nursing to music therapy, allows for a larger range of inputs. In addition, it is worth seeking out and joining forces with those elements of the medical humanities, most usually medical history and ethics, which may be contained within either heritage, museum and archives sections of medical colleges for the former, or in the taught elements of undergraduate or basic and higher specialist training for the latter.

Given that exposure to this area is novel in postgraduate training and CPD, a number of other approaches may be helpful. A key pedagogical element is to view doctors, both in and after formal postgraduate training, as partners in the process, and curricula should be developed drawing on the rich experiences, traditions and cultural engagement of clinicians through appropriate 
enquiry and soundings. ${ }^{4}$ It would also be helpful to have regular introductory courses, using a range of educational methods including online courses, such as that provided by the University of Cape Town (www.futurelearn.com/courses/medicine-and-the-arts).

Working with engaged scholars in the arts and humanities is clearly also a vital element of such programmes but requires consideration and insight into the challenges and rewards of interdisciplinary working, mindful that current publication practices suggest that such engagement remains a minority practice in the medical humanities for both clinicians and scholars in arts and humanities. ${ }^{5,39,40}$

Medical humanities add depth and richness to the practice of medicine. As eloquently outlined in an insightful critique of narrative medicine, the study of medical humanities should provide hinterland and perspective: hinterland as a connection with broader culture and the world beyond the medical school and the hospital, and perspective as an understanding of the place of medicine in society, the historical forces that have shaped it and the challenges it will face in the future. ${ }^{41}$ The possibility to generate a body of clinical faculty and CPD activities represents an enormous opportunity to provide balance and perspective to support clinical practice and professional satisfaction in rapidly changing and stressful healthcare environment. .

\section{Acknowledgements}

We would like to express our gratitude to the foresight and encouragement of Dr Sinéad Murphy, director of postgraduate education, and Dr Ann O'Shaughnessy, RCPI, in supporting the development of this programme of CPD and postgraduate training in medical humanities in the college, as well as members of our interest group in medical humanities, including Dr Elizabeth Barrett, Dr Muiris Houston and Prof Chris Fitzpatrick.

\section{References}

1 Eliot TS. Four quartets. New York: Harcourt, 1943.

2 Peterkin AD, Skorzewska A. Health humanities in postgraduate medical education. Oxford: Oxford University Press, 2018.

3 O'Neill D. Bicycle helmets and the medical humanities. BMJ Opinion 2012. https://blogs.bmj.com/bmj/2012/08/03/desmond oneill-bicycle-helmets-and-the-medical-humanities

4 O'Neill D, Jenkins E, Mawhinney R et al. Rethinking the medical in the medical humanities. Med Humanit 2016:42:109-14.

5 King R, Al-Khabouri ], Kelly BD, O'Neill D. Authorship in the medical humanities: breaking cross-field boundaries or maintaining disciplinary divides? J Med Humanities 2019:[Epub ahead of print].

6 Pellegrino ED. Humanism and the physician. Knoxville: University of Tennessee Press, 1979

7 Moss H, O'Neill D. Medical humanities - serious academic pursuit or doorway to dilettantism? Irish Medical Journal 2012;105:261-2.

8 Medical history without medicine. Journal of the History of Medicine and Allied Sciences 1980;35:5-7.

9 Porter R, Micale MS. Introduction: reflections on psychiatry and its histories. In: Micale MS, Porter R (eds), Discovering the history of psychiatry. New York and Oxford: Oxford University Press, 1994:3-36.

10 Burnham JC. What is medical history? Cambridge: Polity Press, 2005.

11 Sen A. The idea of justice. London: Allen Lane, 2009.

12 Kelly BD. Mental illness, human rights and the law. London: RCPsych Publications, 2016.

13 Duffy RM, Kelly BD. The right to mental healthcare: India moves forward. Br J Psychiatry 2019;214:59-60.

14 Duffy RM, Kelly BD. India's Mental Healthcare Act, 2017: Content, context, controversy. Int J Law Psychiatry 2019;62:169-78.

15 Beresford MJ. Medical reductionism: lessons from the great philosophers. QJM 2010;103:721-4.
16 Harris J. In praise of unprincipled ethics. Journal of Medical Ethics 2003;29:303-6.

17 Beauchamp TL, Childress JF. Principles of biomedical ethics. Oxford: Oxford University Press, 2001.

18 Schneider CE. The practice of autonomy: patients, doctors, and medical decisions. New York: Oxford University Press, 1998.

19 Nash W. Showing that medical ethics cases can miss the point: rewriting short stories as cases. Literature and Medicine 2018;36:190-207.

20 Terry JS, Gogel EL. Poems and patients: the balance of interpretation. Literature and Medicine 1987;6:43-53.

21 Coulehan J (ed), Chekhov's doctors: a collection of Chekhov's medical tales. Kent: The Kent State University Press, 2003.

22 Eliot G. Middlemarch. William Blackwood and Sons, 1871.

23 Stempsey WE. The quarantine of philosophy in medical education: why teaching the humanities may not produce humane physicians. Medicine, Health Care, and Philosophy 1999;2:3-9.

24 Koschorke A. Tyrants writing poetry. Budapest: Central European University Press, 2018.

25 Dans PE. A personal journey using film to teach medical ethics. In: Colt H, Quadrelli S, Lester F (eds), The picture of health: Medical ethics and the movies. Oxford: Oxford University Press, 2015:1-9.

26 Loughlin E, O'Keeffe ST. One man and his dog: Umberto D. Eur Geriatr Med 2019:10:673-4.

27 Beauchamp TL, Childress JF. Principles of biomedical ethics, 7th edn. Oxford: Oxford University Press, 2013.

28 Wynn Owen J, Phillips R, Thorne P et al. Arts, health and wellbeing: Beyond the millennium. London: Royal Society for Public Health, 2013.

29 Stuckey HL, Nobel J. The connection between art, healing, and public health: A review of current literature. American journal of public health 2010;100:254-63.

30 Clift S, Camic PM. Oxford textbook of creative arts, health, and wellbeing: International perspectives on practice, policy and research. Oxford University Press, 2016.

31 Moss H, O'Neill D. What training do artists need to work in healthcare settings? Med Humanit 2009;35:101-5.

32 Moss H, O'Neill D. The role of the curator in modern hospitals: A transcontinental perspective. The journal of medical humanities 2019:40:85-100.

33 Cameron M, Crane N, Ings R, Taylor K. Promoting well-being through creativity: how arts and public health can learn from each other. Perspectives in public health 2013;133:52-9.

34 Anderson DC, Pang SA, O'Neill D, Edelstein EA. The convergence of architectural design and health. Lancet 2018;392:2432-3.

35 Ulrich RS, Zimring C, Zhu $X$ et al. A review of the research literature on evidence-based healthcare design. Herd 2008;1:61-125.

36 Kaptein AA, Meulenberg F, Smyth JM. A breath of fresh air: images of respiratory illness in novels, poems, films, music, and paintings. Journal of health psychology 2015;20:246-58.

37 Kaptein AA, Thong MSY. Portraying a grim illness: lung cancer in novels, poems, films, music, and paintings. Supportive Care Cancer 2018;26:3681-9.

38 Moss H, O'Neill D. The aesthetic and cultural interests of patients attending an acute hospital - a phenomenological study. J Adv Nurs 2014:70:121-9.

39 Russell C, O'Neill D. Ethicists and clinicians: the case for collaboration in the teaching of medical ethics. Irish medical journal 2006:99:25-7.

40 Callard F, Fitzgerald D. Rethinking interdisciplinarity across the social sciences and neurosciences. Palgrave Macmillan, 2015:160.

41 O'Mahony S. Against narrative medicine. Perspect Biol Med 2013:56:611-9.

Address for correspondence: Prof Desmond O'Neill, Royal College of Physicians of Ireland, Kildare Street, Dublin 2, Ireland.

Email: doneill@tcd.ie 